\title{
Long noncoding RNA NEAT1 sponges miR-125a-5p to suppress cardiomyocyte apoptosis via BCL2L12
}

\author{
HONG YAN, HUASHENG LIANG, LIE LIU, DONGLI CHEN and QIANHUAN ZHANG \\ Department of Cardiology, Guangdong Cardiovascular Research Institute, Guangdong General Hospital, \\ Guangdong Academy of Medical Sciences, Guangzhou, Guangdong 510515, P.R. China
}

Received July 29, 2018; Accepted January 30, 2019

DOI: $10.3892 / \mathrm{mmr} .2019 .10095$

\begin{abstract}
Increasing evidence has suggested that long non-coding RNA nuclear paraspeckle assembly transcript 1 (NEAT1) has critical roles in multiple biological processes; however, few studies have reported on its function in heart disease. The present study indicated that NEAT1 expression is markedly downregulated in cardiomyocytes following ischemia/reperfusion injury in vivo and hydrogen peroxide treatment in vitro. Further experiments suggested that ectopic overexpression of NEAT1 suppresses cardiomyocyte apoptosis induced by hydrogen peroxide, as assessed by TUNEL assay and flow cytometry. In addition, using a dual-luciferase reporter assay, NEAT1 was demonstrated to directly interact with microRNA (miR)-125a-5p and overexpression of miR-125a-5p efficiently reversed the stimulatory effect of NEAT1 on B-cell lymphoma-2-like 12 (BCL2L12) expression. Furthermore, the results indicated that NEAT1 inhibits cardiomyocyte apoptosis via regulating the expression of BCL2L12, which appeared to be mediated via miR-125a-5p. In conclusion, the present study suggested that NEAT1 functions as a miR sponge to inhibit cardiomyocyte apoptosis and may be a novel therapeutic target for cardiomyocyte apoptosis-associated heart diseases.
\end{abstract}

\section{Introduction}

Ischemic heart disease (IHD) is a major cause of death and disability worldwide $(1,2)$. Increasing evidence indicates that cardiomyocyte apoptosis is closely associated with the pathogenesis of IHD (3). Although our understanding of the

Correspondence to: Dr Qianhuan Zhang, Department of Cardiology, Guangdong Cardiovascular Research Institute, Guangdong General Hospital, Guangdong Academy of Medical Sciences, 96 Dongchuan Road, Guangzhou, Guangdong 510515, P.R. China

E-mail: zhangqianhuan18@sina.com

Key words: cardiomyocyte apoptosis, long non-coding RNA nuclear paraspeckle assembly transcript 1, microRNA-125a-5p, B-cell lymphoma-2-like 12 molecular components involved in cardiomyocyte apoptosis has rapidly improved, no treatment to inhibit this cellular process is currently available (4). Therefore, it is crucial to further elucidate the molecular mechanisms underlying cardiomyocyte apoptosis and identify effective therapeutic targets for the treatment of IHD.

Long non-coding RNAs (lncRNAs) have been reported to have important roles in the development of cardiovascular diseases (5). LncRNAs are defined as a subgroup of RNAs lacking a protein-coding ability with a length of $>200$ nucleotides (6). Through a variety of mechanisms, lncRNAs are involved in numerous cellular processes, including cell apoptosis $(7,8)$. Furthermore, the dysregulation of certain lncRNAs is associated with the development of various diseases, including IHD (9). Mounting evidence has indicated that lncRNAs may interact with microRNAs (miRNAs/miRs) and function as competing endogenous RNAs (ceRNAs) to regulate gene expression (10).

Nuclear paraspeckle assembly transcript 1 (NEAT1), a structural component of paraspeckle, is a newly identified nuclear-restricted lncRNA and acts as a transcriptional regulator for numerous genes (11). Aberrant expression of NEAT1 has been reported in multiple different types of tumour, including lung cancer and acute promyelocytic leukaemia $(12,13)$. In addition, studies have indicated that NEAT1 is involved in the pathogenesis of diverse diseases by regulating the activity of miRNAs $(14,15)$; however, whether NEAT1 is involved in the regulation of cardiomyocyte apoptosis has remained elusive.

The present study identified that NEAT1 is downregulated in murine heart tissues subjected to ischemia/reperfusion (I/R) injury and in $\mathrm{H}_{2} \mathrm{O}_{2}$-induced cardiomyocytes. Further in vitro study confirmed that ectopic overexpression of NEAT1 inhibits $\mathrm{H}_{2} \mathrm{O}_{2}$ treatment-induced cardiomyocyte apoptosis. Furthermore, a search of the starBase database v2.0 (http://starbase.sysu.edu.cn) indicated that miR-125a-5p binds to NEAT1. It was also revealed that NEAT1 serves as an miRNA sponge to sequester miR-125a-5p and increase the expression of apoptosis repressor gene B-cell lymphoma-2-like 12 (BCL2L12) in cardiomyocytes. The present study provides novel insight into the roles of NEAT1 in cardiomyocyte apoptosis, which may be applied in the treatment of cardiomyocyte apoptosis-associated heart diseases. 


\section{Materials and methods}

Animal experiment. In total, 20 adult male C57BL/6 mice (age, 8 weeks; weight, 18-20 g) were purchased from Shanghai Biomodel Organism Science \& Technology Development Co., Ltd. (Shanghai, China). Mice had access to food and water, maintained under constant temperature at $25 \pm 1^{\circ} \mathrm{C}$, $55 \pm 5 \%$ humidity, under a 12 -h light/dark cycle. All animal experiments in this study were approved by the Animal Ethics Committee of Guangdong Cardiovascular Research Institute (Guangzhou, China). I/R surgery was performed as previously described (16).

Cardiomyocyte culture and treatment. Cardiomyocytes were isolated from the ventricular myocardium of 2-day-old Sprague Dawley rats, as described previously (17). In brief, hearts were dissected and cells were subsequently dispersed through enzymatic digestion. Cultured neonatal rat cardiomyocytes were incubated in culture medium (control group) or treated with $\mathrm{H}_{2} \mathrm{O}_{2}$ at $100 \mu \mathrm{M}$ for $0,6,12$ and 24 h. $\left(\mathrm{H}_{2} \mathrm{O}_{2}\right.$ group). Cell apoptosis was detected when cardiomyocytes were treated with $100 \mu \mathrm{M}$ for $24 \mathrm{~h}$. Cells were cultured in Dulbecco's Modified Eagle's Medium (Gibco; Thermo Fisher Scientific, Inc., Waltham, MA, USA) supplemented with $10 \%$ FBS (HyClone; GE Healthcare Life Sciences, Logan, UT, USA) and antibiotics (100 U/ml penicillin and $100 \mathrm{mg} / \mathrm{ml}$ streptomycin). Cells were incubated at $37^{\circ} \mathrm{C}$ with $5 \% \mathrm{CO}_{2}$.

Cardiomyocyte apoptosis by terminal deoxynucleotidyl transferase-mediated dUTP nick-end labeling (TUNEL) staining and flow cytometry. Cell apoptosis was determined by a TUNEL assay using $1 \times 10^{5}$ cells (DeadEnd ${ }^{\mathrm{TM}}$ Colorimetric TUNEL System; Promega Corporation, Madison, WI, USA) according to the manufacturer's protocol. In addition, $1 \times 10^{5}$ cardiomyocytes were stained with Annexin V-fluorescein isothiocyanate and propidium iodide (BD Pharmingen; Becton, Dickinson and Company, Franklin, Lakes, NJ, USA), and then subjected to flow cytometry (CellQuest Pro software (version 5.1; Becton, Dickinson and Company) to determine apoptosis.

Dual-luciferase reporter assay. Luciferase reporter assay was performed according to a published protocol (18). In brief, the wild-type (wt) and mutant (mut) miR-125a-5p binding site sequences in the NEAT1 3'-untranslated region were used to generate Luc-NEAT1-wt vector and Luc-NEAT1-mut vector using pGL3 as backbone (Promega Corporation). For reporter assays, $1 \times 10^{3} 293$ cells (American Type Culture Collection, Manassas, VA, USA) were transfected using Lipofectamine ${ }^{\circledR} 2000$ (Invitrogen; Thermo Fisher Scientific, Inc.) with either NEAT1-wt or NEAT1-mut construct $(500 \mathrm{ng})$ with and without miR-125a-5p mimic. The miR-125a-5p mimic and miR-negative control (NC) were transfected at a concentration of $0.2 \mathrm{nmol}$ per well and were purchased from Guangzhou RiboBio Co., Ltd. (Guangzhou, China). Luciferase activity was measured $48 \mathrm{~h}$ after transfection using the Dual-Luciferase Reporter Assay System (pRL vector; Promega Corporation). The Renilla luciferase activity was normalized to the firefly luciferase activity in the corresponding well.
Reverse transcription-quantitative polymerase chain reaction $(R T-q P C R)$. Total RNA was isolated using TRIzol ${ }^{\circledR}$ reagent (Invitrogen; Thermo Fisher Scientific, Inc.) according to the manufacturer's protocol. RNA was reverse transcribed using the PrimeScript RT Reagent Kit (Invitrogen; Thermo Fisher Scientific, Inc.) at $37^{\circ} \mathrm{C}$ for $15 \mathrm{~min}$ and at $85^{\circ} \mathrm{C}$ for $5 \mathrm{sec}$ and qPCR was performed using SYBR Premix Ex Taq (Takara Biotechnology Co., Ltd., Dalian, China) with 35 cycles of $95^{\circ} \mathrm{C}$ for $1 \mathrm{~min} ; 55^{\circ} \mathrm{C}$ for $1 \mathrm{~min}$ and $72^{\circ} \mathrm{C}$ for $1 \mathrm{~min}$ ). The relative mRNA expression level was calculated using the comparative cycle quantification $\left(2^{-\Delta \Delta C q}\right)$ method (19) with GAPDH as the endogenous control to normalize the data. The primers used for qRT-PCR were as follows: NEAT1 forward, 5'-TGGCTAGCT CAGGGCTTCAG-3' and reverse, 5'-TCTCCTTGCCAAGCT TCCTT-3'; GAPDH forward, 5'-CGCTCTCTGCTCCTCCTG TTC-3' and reverse, 5'-ATCCGTTGACTCCGACCTTCAC-3'.

RNA immunoprecipitation (RIP) assays. RIP experiments were performed by using a Magna RIP RNA-Binding Protein Immunoprecipitation Kit (EMD Millipore, Billerica, MA, USA) according to the manufacturer's protocol. In brief, cells were lysed in RIP lysis buffer, following incubation with RIP buffer containing magnetic beads conjugated with anti-argonaute 2, RISC catalytic component (AGO2) antibody. Anti-small nuclear ribonucleoprotein U1 subunit 70 was used as a positive control for the RIP procedure. The co-precipitated RNAs were detected by RT-qPCR analysis performed as above-mentioned. The primers used for miR-125a-5p was as follows: Forward: 5'-ACGGTGCTGGATGTGGCCTTT-3', reverse: 5'-GGCCAACCGCGAGAAGATGTTTTTTTTT-3'.

Western blot analysis. Western blot analyses were performed using standard methods (20). The primary antibodies used were as follows: Anti-BCL2L12 (1:800 dilution; Abcam, Cambridge, MA, USA) and anti-tubulin (1:1,000 dilution; Santa Cruz Biotechnology, Inc., Dallas, TX, USA). The signals were detected by enhanced chemiluminescence (Pierce; Thermo Fisher Scientific, Inc.).

Statistical analysis. All data are expressed as the mean \pm standard error. Differences between groups were analysed using a two-tailed Student's t-test and One-way analysis of variance. $\mathrm{P}<0.05$ was considered to indicate a statistically significant difference.

\section{Results}

NEATl expression was downregulated in heart tissues during $I / R$ injury and $\mathrm{H}_{2} \mathrm{O}_{2}$-induced cardiomyocytes. To investigate the expression and role of NEAT1 in I/R-injured myocardium, a mouse model of I/R injury was established. The expression of NEAT1 in the heart tissues of mice with or without $\mathrm{I} / \mathrm{R}$ injury was then assessed by RT-qPCR. As presented in Fig. 1A, the RT-qPCR analysis revealed that the expression of NEAT1 was significantly downregulated in ischemic myocardium compared with sham-operated controls.

Additionally, the primary cultured cardiomyocytes were subjected to $\mathrm{H}_{2} \mathrm{O}_{2}$ treatment in vitro. Consistent with the result obtained in the I/R-injured hearts, the expression of NEAT1 was also downregulated in cardiomyocytes following $\mathrm{H}_{2} \mathrm{O}_{2}$ 

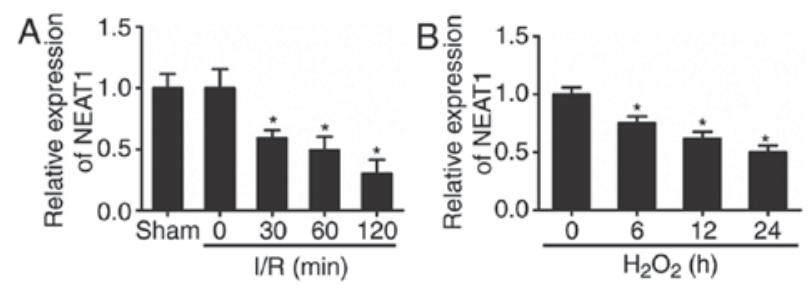

Figure 1. NEAT1 expression in I/R injured hearts and $\mathrm{H}_{2} \mathrm{O}_{2}$-induced cardiomyocytes. (A) Expression levels of NEAT1 following I/R injury. Mice were subjected to I/R injury for the indicated durations and the expression of NEAT1 was assayed by RT-qPCR ( $\mathrm{n}=6$ mice for each time point). ${ }^{*} \mathrm{P}<0.05 \mathrm{vs}$. sham. (B) Cardiomyocytes were treated with $100 \mathrm{mM} \mathrm{H}_{2} \mathrm{O}_{2}$ for the indicated durations and NEAT1 levels were determined by RT-qPCR. Three independent experiments were performed, and the data are presented as the mean \pm standard deviation. ${ }^{*} \mathrm{P}<0.05$ vs. 0 h. RT-qPCR, reverse transcription-quantitative polymerase chain reaction; NEAT1, nuclear paraspeckle assembly transcript 1; I/R, ischemia/reperfusion.

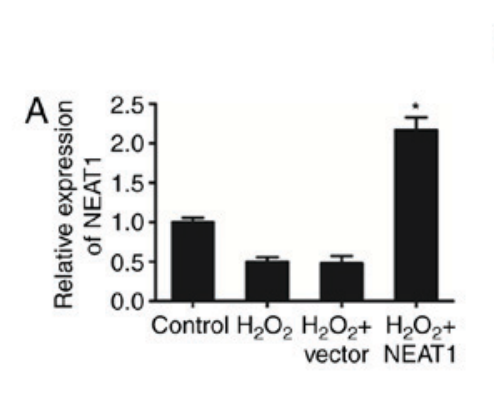

B Control $\begin{array}{llll}\mathrm{H}_{2} \mathrm{O}_{2} & \mathrm{H}_{2} \mathrm{O}_{2}+ & \mathrm{H}_{2} \mathrm{O}_{2}+ \\ \text { vector } & \mathrm{NEAT1}\end{array}$
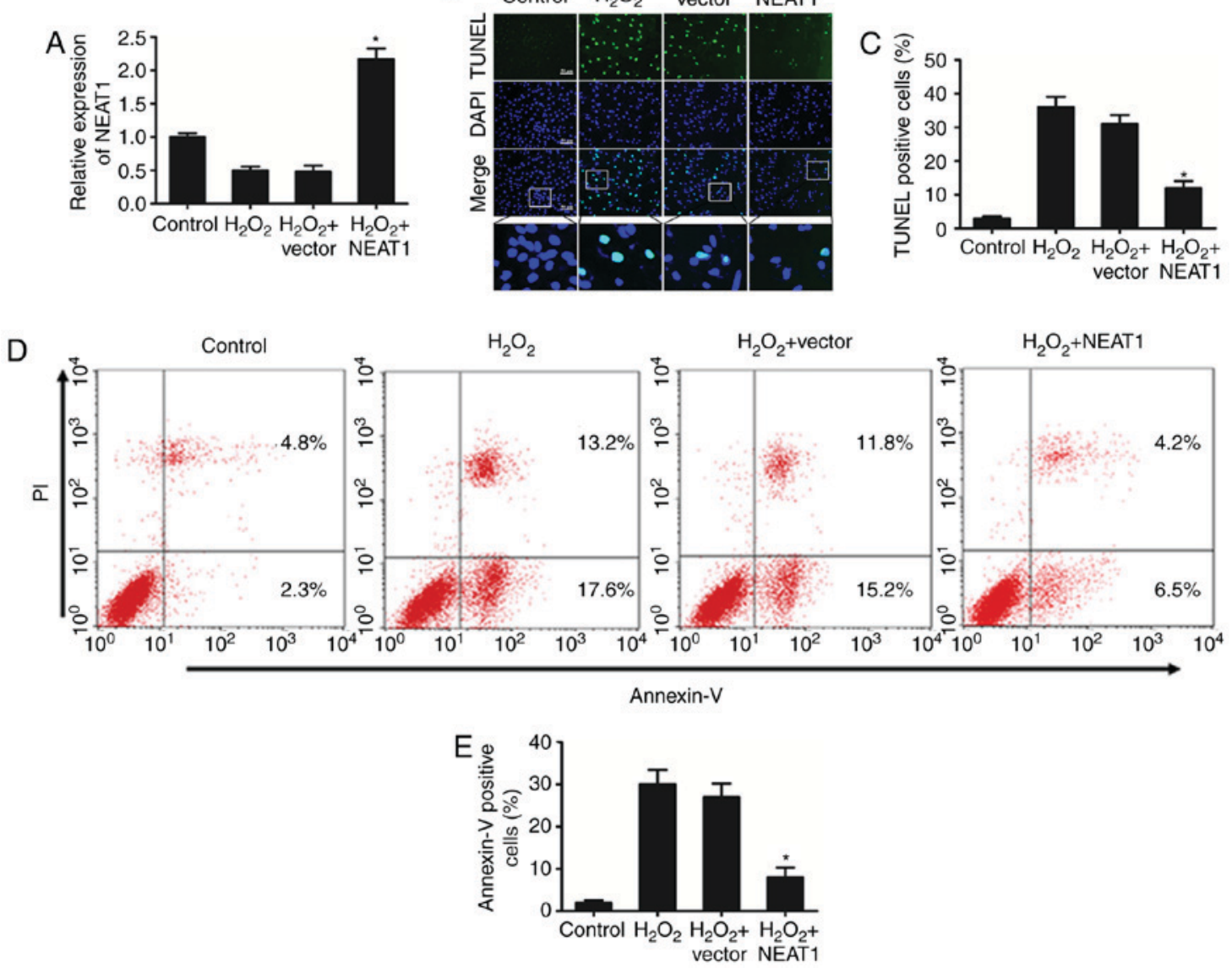

Figure 2. Overexpression of NEAT1 inhibits $\mathrm{H}_{2} \mathrm{O}_{2}$-induced cardiomyocyte apoptosis. (A) Following transfection with a NEAT1 overexpression plasmid, the cardiomyocytes were treated with $100 \mathrm{mM} \mathrm{H}_{2} \mathrm{O}_{2}$ for $24 \mathrm{~h}$. Subsequently, the expression of NEAT1 was detected by reverse transcription-quantitative polymerase chain reaction. Three independent experiments were performed, and the data are presented as the mean \pm standard deviation. ${ }^{*} \mathrm{P}<0.05$ vs. control group. A TUNEL assay was used to (B) identify apoptotic cardiomyocytes, and (C) the percentage of TUNEL-positive apoptotic cardiomyocytes was quantitatively determined. Three independent experiments were performed, and the data are presented as the mean \pm standard deviation. " $\mathrm{P}<0.05$ vs. control group. Bar, $50 \mu \mathrm{m}$. (D) Apoptosis analysis flow plots and (E) the percentage of the Annexin-V-positive cell population was calculated. Three independent experiments were performed, and the data are presented as the mean \pm standard deviation. ${ }^{*} \mathrm{P}<0.05$ vs. control group. NEAT1, nuclear paraspeckle assembly transcript 1 ; TUNEL, terminal deoxynucleotidyl transferase-mediated dUTP nick-end labeling; PI, propidium iodide.

treatment (Fig. 1B). These results indicated that the expression of NEAT1 was obviously decreased in I/R-injured hearts and $\mathrm{H}_{2} \mathrm{O}_{2}$-treated cardiomyocytes.

Enhanced expression of $\mathrm{NEATl}$ attenuated $\mathrm{H}_{2} \mathrm{O}_{2}$-induced cardiomyocyte apoptosis. To explore the potential biological function of NEAT1 in cardiomyocyte apoptosis, the cultured cardiomyocytes were transfected with a plasmid encoding
NEAT1. As presented in Fig. 2A, cells transfected with NEAT1 expression plasmid exhibited obviously increased expression levels of NEAT1 compared with those in the control and vector groups. The TUNEL assay demonstrated that overexpression of NEAT1 inhibited cardiomyocyte apoptosis in response to $\mathrm{H}_{2} \mathrm{O}_{2}$ treatment compared with that in the control and vector groups (Fig. 2B and C). Consistent with these results, the flow cytometry data also indicated that overexpression 


\section{A NEAT1 wt 5'-ggg AGG-AAGTGGCTAGCTCAGGGc-3' ||| |: | | | ||||||| miR-125a-5p 3'-agtgTCCAATTTCCCA - - GAGTCCCt-5' NEAT1 mut $\quad 5^{\prime}$-ggg CAT-ACGTATCGAGAGTCTTAC-3'}
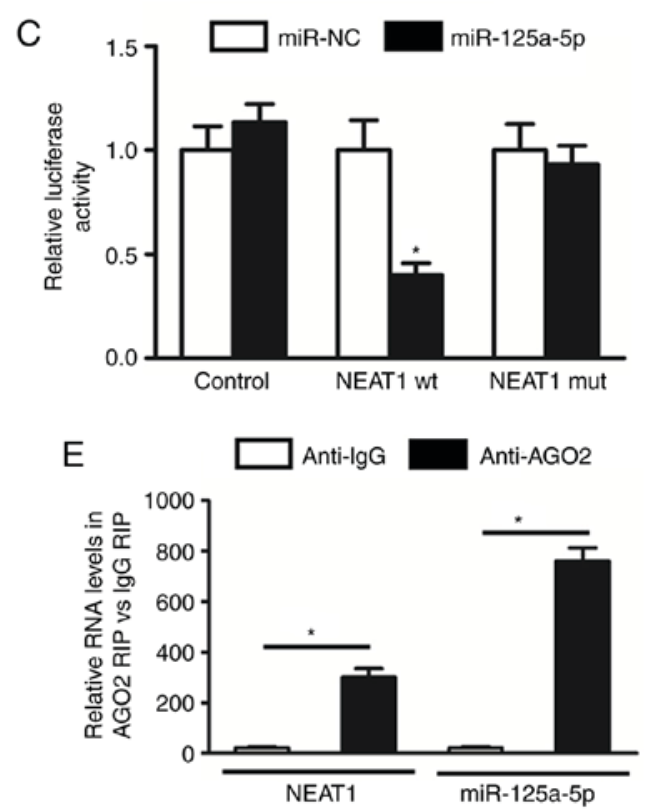

\section{B}
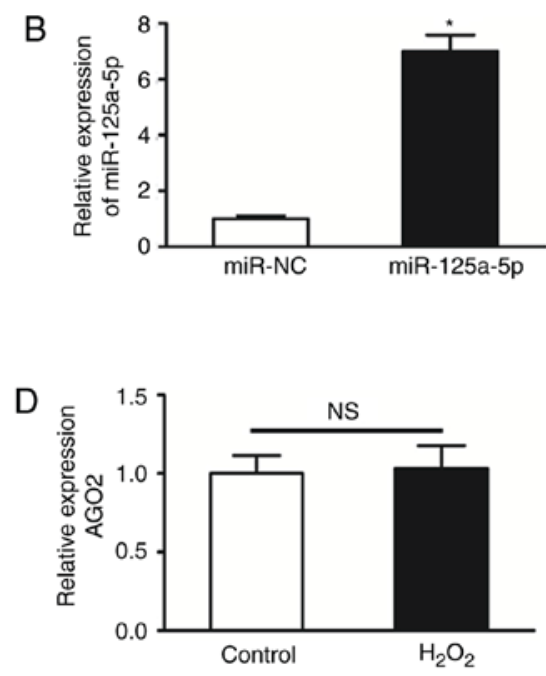

$\mathrm{F}$
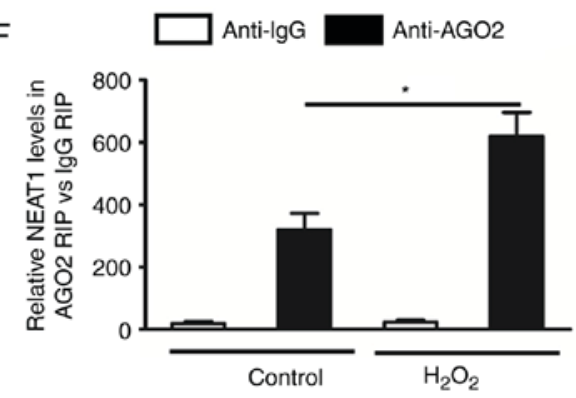

Figure 3. NEAT1 is targeted by miR-125a-5p in an AGO2-dependent manner. (A) Putative complementary sites between NEAT1 and miR-125a-5p. Mutation was generated in the NEAT1 sequence at the complementary sites of miR-125a-5p, as indicated. (B) The miR-125a-5p expression was increased in 293 cells transfected with miR-125a-5p mimics. (C) Luciferase activity in 293 cells co-transfected with miR-125a-5p mimics or NC, and luciferase reporters containing no insert (control), NEAT1 wt or NEAT1 mut. Three independent experiments were performed, and the data are presented as the mean \pm standard deviation. "P $<0.05$ vs. miR-NC group. (D) The expression of AGO2 was detected in cardiomyocytes subjected to $\mathrm{H}_{2} \mathrm{O}_{2}$ treatment. (E) A RIP assay was performed to confirm whether NEAT1 and miR-125a-5p directly bind to AGO2 in cardiomyocytes. (F) The RIP experiments were performed under $\mathrm{H}_{2} \mathrm{O}_{2}$ treatment, and the expression of NEAT1 was then assessed by reverse transcription-quantitative polymerase chain reaction analysis. Three independent experiments were performed, and the data are shown as the mean \pm standard deviation. "P $<0.05$ vs IgG. NEAT1, nuclear paraspeckle assembly transcript 1 ; wt, wild-type; miR, microRNA; mut, mutant; NC, negative control; AGO2, argonaute 2, RISC catalytic component; RIP, RNA immunoprecipitation.

of NEAT1 reduced $\mathrm{H}_{2} \mathrm{O}_{2}$-induced cardiomyocyte apoptosis when compared with that in the control and vector groups (Fig. 2D and E).

NEAT1 is targeted by miR-125a-5p in an AGO2-dependent manner. To investigate the molecular mechanisms of NEAT1 in regulating cardiomyocyte apoptosis, the present study explored which miRNAs are able to bind to NEAT1. A bioinformatics prediction with Starbase v2.0 software revealed that miR-125a-5p has complementary base pairing with NEAT1. Subsequently, a luciferase vector containing the binding sequence of NEAT1, Luc-NEAT1-wt, and its mutated form, Luc-NEAT1-mut, were constructed (Fig. 3A). It was confirmed that the expression of miR-125a-5p was increased in 293 cells transfected with miR-125a-5p mimics (Fig. 3B). As presented in Fig. 3C, the luciferase activity was obviously inhibited in the presence of miR-125a-5p and was recovered when the binding site for miR-125a-5p was mutated. These results confirmed that NEAT1 directly interacts with miR-125a-5p.

Subsequently, to confirm the pivotal roles of miR-125a-5p in this effect, an RIP assay was performed on AGO2, which is the core component of the RNA-induced silencing complex. First, it was indicated that $\mathrm{H}_{2} \mathrm{O}_{2}$ treatment had no effect on the expression of AGO2 in cardiomyocytes (Fig. 3D). Furthermore, the RIP assay indicated that NEAT1 and miR-125a-5p were preferentially enriched in the AGO2-containing miRNA ribonucleoprotein complex when compared with the immunoglobulin $\mathrm{G}$ immunoprecipitates (Fig. 3E). In addition, the binding of NEAT1 to AGO2 in cardiomyocytes was enhanced under $\mathrm{H}_{2} \mathrm{O}_{2}$ treatment (Fig. 3F).

NEAT1 regulates cardiomyocyte apoptosis through targeting miR-125a-5p and BCL 2 L12. To investigate the role of $\mathrm{miR}-125 \mathrm{a}-5 \mathrm{p}$ in cardiomyocyte apoptosis, the expression of miR-125a-5p was examined in I/R-injured mouse hearts and in $\mathrm{H}_{2} \mathrm{O}_{2}$ treated cardiomyocytes. The results indicated that the expression levels of miR-125a-5p were significantly upregulated in I/R-injured myocardium and in $\mathrm{H}_{2} \mathrm{O}_{2}$-induced cardiomyocytes (Fig. 4A and B). The effects of miR-125a-5p on cardiomyocyte apoptosis following $\mathrm{H}_{2} \mathrm{O}_{2}$ treatment were then investigated. The expression level of miR-125a-5p was examined following transfection with miR-125a-5p inhibitor and mimic (Fig. 4C). As expected, the results demonstrated that knockdown of miR-125a-5p significantly suppressed cardiomyocyte apoptosis induced by $\mathrm{H}_{2} \mathrm{O}_{2}$ (Fig. 4D and E).

A previous study has demonstrated that miR-125a-5p induces apoptosis in colon cancer by targeting BCL2L12 (21). 


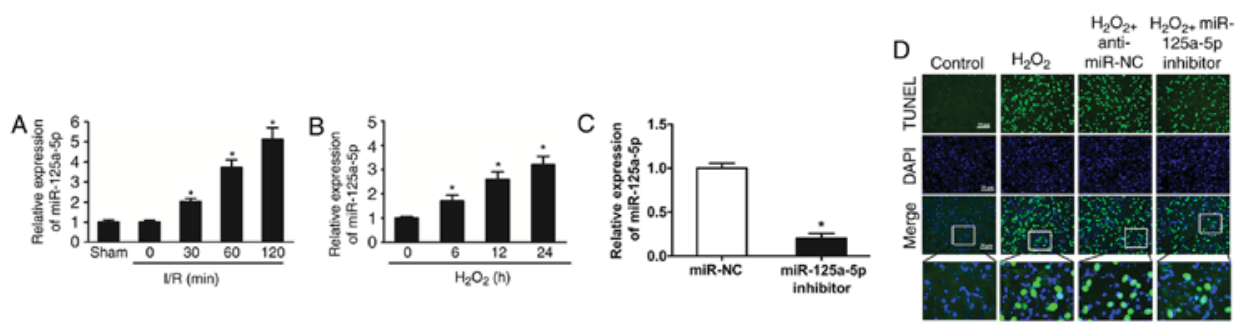

E

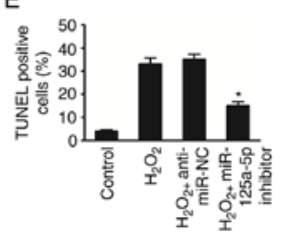

F

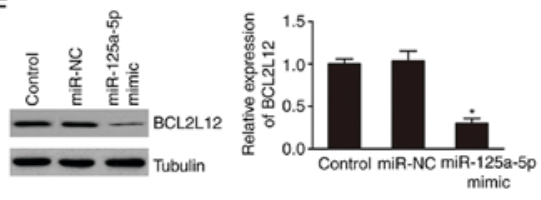

G

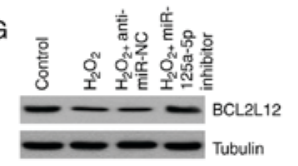

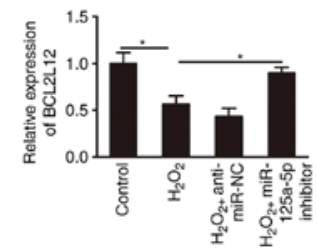

H

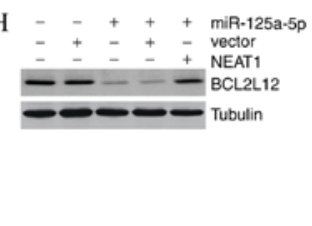

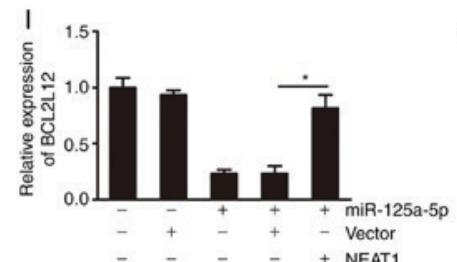
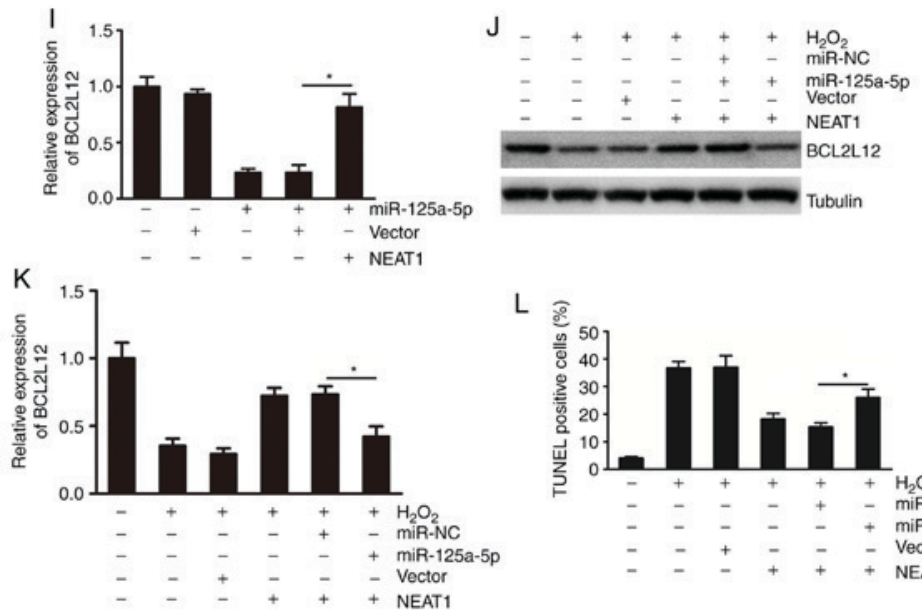

L
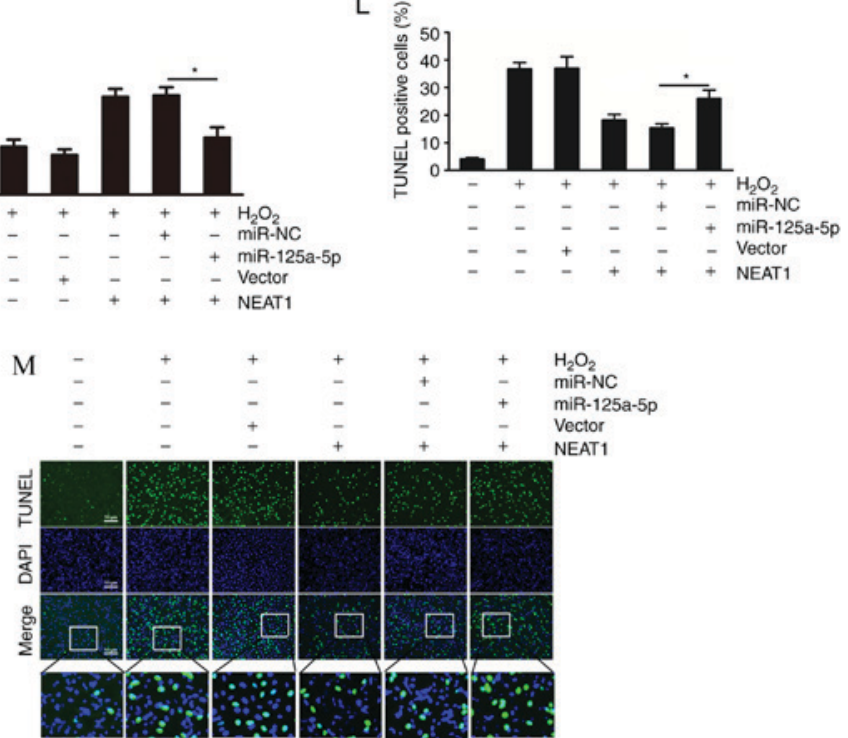

Figure 4. NEAT1 regulates cardiomyocyte apoptosis through miR-125a-5p and BCL2L12. (A) Mice were subjected to I/R injury and the expression of miR-125a-5p was determined after the indicated periods of time ( $\mathrm{n}=6$ mice for each time-point). * $\mathrm{P}<0.05$ vs. sham. (B) Cardiomyocytes were treated with $100 \mathrm{mM} \mathrm{H}_{2} \mathrm{O}_{2}$ and miR-125a-5p levels were detected by reverse transcription-quantitative polymerase chain reaction. Three independent experiments were performed, and the data are shown as the mean \pm standard deviation. ${ }^{*} \mathrm{P}<0.05$ vs. $0 \mathrm{~h}$. (C) miR-125a-5p expression level was decreased in cardiomyocytes transfected with miR-125a-5p inhibitor. ${ }^{*} \mathrm{P}<0.05$ vs. miR-NC. (D) TUNEL assay was used to identify apoptotic cardiomyocytes, and (E) the percentage of apoptotic cardiomyocytes was quantitatively determined. Three independent experiments were performed, and the data are shown as the mean \pm standard deviation. ${ }^{*} \mathrm{P}<0.05$ vs. control group. Bar, $50 \mu \mathrm{m}$. (F) miR-125a-5p mimics or NC were used to transfect cardiomyocytes. The expression of BCL2L12 was detected by immunoblot analysis. Three independent experiments were performed, and the data are shown as the mean \pm standard deviation. ${ }^{*} \mathrm{P}<0.05$ vs. control group. (G) BCL2L12 levels in different groups were detected by immunoblot analysis. Three independent experiments were performed, and the data are shown as the mean \pm standard deviation. ${ }^{*} \mathrm{P}<0.05$ vs. control group. (H and I) Cardiomyocytes were transfected with miR-125a-5p mimics, NEAT1, and BCL2L12 levels were determined by immunoblot analysis. Three independent experiments were performed, and the data are shown as the mean \pm standard deviation. "P<0.05 vs. control group. ( $\mathrm{J}$ and K) BCL2L12 expression was analysed by immunoblot in the different groups. Three independent experiments were performed, and the data are shown as the mean \pm standard deviation. ${ }^{*} \mathrm{P}<0.05$ vs. control group. (L) The percentage of apoptotic cardiomyocytes was quantitatively determined from (M) TUNEL analysis. Three independent experiments were performed, and the data are shown as the mean standard deviation. ${ }^{*} \mathrm{P}<0.05$ vs. control group. Bar, $50 \mu \mathrm{m}$. miR, microRNA; I/R, ischemia/reperfusion; NC, negative control; TUNEL, terminal deoxynucleotidyl transferase-mediated dUTP nick-end labeling; BCL2L12, B-cell lymphoma-2-like 12; NEAT1, nuclear paraspeckle assembly transcript 1. 
To explore whether miR-125a-5p is responsible for the regulation of BCL2L12 in cardiomyocyte apoptosis induced by $\mathrm{H}_{2} \mathrm{O}_{2}$, the expression levels of BCL2L12 following overexpression or silencing of miR-125a-5p in the cultured cardiomyocytes was examined. The results indicated that ectopic overexpression of miR-125a-5p in cardiomyocytes led to a reduction in BCL2L12 levels (Fig. 4F). In addition, knockdown of miR-125a-5p abolished the decrease in BCL2L12 levels in cardiomyocytes following $\mathrm{H}_{2} \mathrm{O}_{2}$ treatment (Fig. 4G).

Subsequently, the association between NEAT1, miR-125a-5p and BCL2L12 in cardiomyocytes was explored. As presented in Fig. $4 \mathrm{H}$ and I, overexpression of NEAT1 attenuated the inhibitory effect of miR-125a-5p on BCL2L12 levels. Notably, overexpression of miR-125a-5p abolished the effect of NEAT1 on BCL2L12 expression and apoptosis under $\mathrm{H}_{2} \mathrm{O}_{2}$ treatment (Fig. 4J-M). Overall, these results suggest that NEAT1 impairs the miR-125a-5p/BCL2L12 interaction in cardiomyocytes to inhibit $\mathrm{H}_{2} \mathrm{O}_{2}$-induced apoptosis.

\section{Discussion}

Cardiomyocyte apoptosis is an important contributor to the development of myocardial infarction and heart failure (22). Although evidence has indicated the upregulation of lncRNA NEAT1 in numerous cancer types $(23,24)$, its role in heart diseases has remained elusive. The present study indicated that ectopic expression of NEAT1 inhibited apoptosis of cultured cardiomyocytes subjected to $\mathrm{H}_{2} \mathrm{O}_{2}$ treatment. The molecular mechanisms by which NEAT1 regulates $\mathrm{H}_{2} \mathrm{O}_{2}$-induced cardiomyocyte apoptosis were then investigated, revealing that NEAT1 functions as a ceRNA to upregulate BCL2L12 expression by sponging miR-125a-5p, which consequently leads to cardiomyocyte apoptosis.

Emerging evidence has revealed that certain lncRNAs are crucial factors in the development of various diseases (25-27). Recent studies have indicated that lncRNAs also contribute to the progression of various types of heart disease (28-30). For instance, lncRNA FTX transcript XIST regulator was reported to suppress cell apoptosis via targeting miR-29b-1-5p and BCL2L12 in cardiomyocytes (16). Zhou et al (29) indicated that lncRNA myocardial infarction associated transcript functions as a ceRNA to upregulate death associated protein kinase 2 by sponging miR-22-3p in diabetic cardiomyopathy. In the present study, the expression of 1ncRNA NEAT1 was significantly downregulated in the myocardium of a mouse model of I/R-induced myocardial injury; however, these data are not in accordance with the recent data from the work of Ma et al (31), which NEAT1 was markedly increased rats following I/R. The distinction could be explained by the potential differential expression of lncRNAs in ischemic rat hearts compared with ischemic mice hearts. Additionally, the previous study was investigating high glucose damage, whereas hypoxia injury was the focus of the present study. Furthermore, NEAT1 was observed to be significantly downregulated in $\mathrm{H}_{2} \mathrm{O}_{2}$-treated cardiomyocytes in the current study, and ectopic overexpression of NEAT1 inhibited $\mathrm{H}_{2} \mathrm{O}_{2}$-induced cardiomyocyte apoptosis in vitro. However, further in vivo investigations are required to verify whether NEAT1 suppresses cardiomyocyte apoptosis in heart tissue.
Regulatory interactions exist between lncRNAs and miRNAs $(10,32)$. LncRNAs may function as ceRNAs and/or molecular sponges to modulate the effects of miRNA. For instance, knockdown of lncRNA $\mathrm{X}$ inactive specific transcript has been reported to exert tumor-suppressive functions in human glioblastoma stem cells by increasing miR-152 levels (33). Based on this known regulatory mechanism and the present results, it may be hypothesized that NEAT1 acts as a ceRNA in cardiomyocytes. In support of this notion, the present results validated the direct binding ability miR-125a-5p to NEAT1, predicted by bioinformatics analysis, and confirmed in luciferase reporter and RIP assays. Further functional assays demonstrated that NEAT1 increased the levels of the miR-125a-5p target gene BCL2L12 by competitively 'sponging' miR-125a-5p in cardiomyocytes, which may be a crucial molecular mechanism involved in the regulation of $\mathrm{H}_{2} \mathrm{O}_{2}$-induced cardiomyocyte apoptosis.

In conclusion, the present study indicated that lncRNA NEAT1 is downregulated in I/R-injured mouse hearts and may function as a ceRNA to upregulate BCL2L12 expression by sponging miR-125a-5p, consequently contributing to cardiomyocyte apoptosis, which is involved in the pathogenesis of ischemic heart disease.

\section{Acknowledgements}

Not applicable.

\section{Funding}

This study was supported by grants from the National Natural Science Foundation of China (grant no. 81470505) and the Province natural science fund of Guangdong (grant no. 2014A030313001).

\section{Availability of data and materials}

Not applicable.

\section{Authors' contributions}

HY designed and revised the manuscript. QZ contributed to the analysis and interpretation of data, and wrote the manuscript. HL, LL and DC performed the experiments. All authors have read and approved the final manuscript.

\section{Ethics approval and consent to participate}

Animal experiments were performed according to the protocol approved by the Animal Care Committee of Guangdong Cardiovascular Research Institute (Guangzhou, China).

\section{Patient consent for publication}

Not applicable.

\section{Competing interests}

The authors declare that they have no competing interests. 


\section{References}

1. Hausenloy DJ and Yellon DM: Targeting myocardial reperfusion injury-the search continues. N Engl J Med 373: 1073-1075, 2015.

2. Writing Group Members; Mozaffarian D, Benjamin EJ, Go AS, Arnett DK, Blaha MJ, Cushman M, Das SR, de Ferranti S, Després JP, et al: Heart disease and stroke statistics-2016 update: A report from the american heart association. Circulation 133 e38-e360, 2016.

3. Kajstura J, Cheng W, Reiss K, Clark WA, Sonnenblick EH, Krajewski S, Reed JC, Olivetti G and Anversa P: Apoptotic and necrotic myocyte cell deaths are independent contributing variables of infarct size in rats. Lab Invest 74: 86-107, 1996.

4. Haunstetter A and Izumo S: Toward antiapoptosis as a new treatment modality. Circ Res 86: 371-376, 2000.

5. Sallam T, Sandhu J and Tontonoz P: Long noncoding RNA discovery in cardiovascular disease: Decoding form to function. Circ Res 122: 155-166, 2018.

6. Mercer TR, Dinger ME and Mattick JS: Long non-coding RNAs: Insights into functions. Nat Rev Genet 10: 155-159, 2009.

7. Khaitan D, Dinger ME, Mazar J, Crawford J, Smith MA, Mattick JS and Perera RJ: The melanoma-upregulated long noncoding RNA SPRY4-IT1 modulates apoptosis and invasion. Cancer Res 71: 3852-3862, 2011.

8. Ginger MR, Shore AN, Contreras A, Rijnkels M, Miller J, Gonzalez-Rimbau MF and Rosen JM: A noncoding RNA is a potential marker of cell fate during mammary gland development. Proc Natl Acad Sci USA 103: 5781-5786, 2006.

9. Saha P, Verma S, Pathak RU and Mishra RK: Long noncoding RNAs in mammalian development and diseases. Adv Exp Med Biol 1008: 155-198, 2017.

10. Juan L, Wang G, Radovich M, Schneider BP, Clare SE, Wang Y and Liu Y: Potential roles of microRNAs in regulating long intergenic noncoding RNAs. BMC Med Genomics 6 (Suppl 1): S7, 2013.

11. Clemson CM, Hutchinson JN, Sara SA, Ensminger AW, Fox AH, Chess A and Lawrence JB: An architectural role for a nuclear noncoding RNA: NEAT1 RNA is essential for the structure of paraspeckles. Mol Cell 33: 717-726, 2009.

12. Qi L, Liu F, Zhang F, Zhang S, Lv L, Bi Y and Yu Y: 1ncRNA NEAT1 competes against let-7a to contribute to non-small cell lung cancer proliferation and metastasis. Biomed Pharmacother 103: 1507-1515, 2018.

13. Zeng C, Xu Y, Xu L, Yu X, Cheng J, Yang L, Chen S and Li Y Inhibition of long non-coding RNA NEAT1 impairs myeloid differentiation in acute promyelocytic leukemia cells. BMC Cancer 14: 693, 2014

14. Tu J, Zhao Z, Xu M, Lu X, Chang L and Ji J: NEAT1 upregulates TGF- $\beta 1$ to induce hepatocellular carcinoma progression by sponging hsa-mir-139-5p. J Cell Physiol 233: 8578-8587, 2018.

15. Chen Y, Qiu J, Chen B, Lin Y, Chen Y, Xie G, Qiu J, Tong H and Jiang D: Long non-coding RNA NEAT1 plays an important role in sepsis-induced acute kidney injury by targeting miR-204 and modulating the NF-kappaB pathway. Int Immunopharmacol 59 252-260, 2018

16. Long B, Li N, Xu XX, Li XX, Xu XJ, Guo D, Zhang D, Wu ZH and Zhang SY: Long noncoding RNA FTX regulates cardiomyocyte apoptosis by targeting miR-29b-1-5p and Bcl212. Biochem Biophys Res Commun 495: 312-318, 2018.

17. Tsybouleva N, Zhang L, Chen S, Patel R, Lutucuta S, Nemoto S, DeFreitas G, Entman M, Carabello BA, Roberts R and Marian AJ Aldosterone, through novel signaling proteins, is a fundamental molecular bridge between the genetic defect and the cardiac phenotype of hypertrophic cardiomyopathy. Circulation 109 . 1284-1291, 2004.
18. Bian Z, Jin L, Zhang J, Yin Y, Quan C, Hu Y, Feng Y, Liu H, Fei B, Mao Y, et al: LncRNA-UCA1 enhances cell proliferation and 5-fluorouracil resistance in colorectal cancer by inhibiting miR-204-5p, Sci Re 6: 23892, 2016.

19. Livak KJ and Schmittgen TD: Analysis of relative gene expression data using real-time quantitative PCR and the 2(-Delta Delta $\mathrm{C}(\mathrm{T})$ ) method. Methods 25: 402-408, 2001

20. Liu H, Hu J, Pan H, Luo D, Huang M and Xu W: CSN5 Promotes hepatocellular carcinoma progression by SCARA5 inhibition through suppressing $\beta$-catenin ubiquitination. Dig Dis Sci 63: 155-165, 2018.

21. Tong Z, Liu N, Lin L, Guo X, Yang D and Zhang Q: miR-125a-5p inhibits cell proliferation and induces apoptosis in colon cancer via targeting BCL2, BCL2L12 and MCL1. Biomed Pharmacother 75: 129-136, 2015.

22. Narula J, Pandey P, Arbustini E, Haider N, Narula N, Kolodgie FD, Dal Bello B, Semigran MJ, Bielsa-Masdeu A, Dec GW, et al: Apoptosis in heart failure: Release of cytochrome c from mitochondria and activation of caspase-3 in human cardiomyopathy. Proc Natl Acad Sci USA 96: 8144-8149, 1999.

23. Liu Y, Wang Y, Fu X and Lu Z: Long non-coding RNA NEAT1 promoted ovarian cancer cells' metastasis through regulation of miR-382-3p/ROCK1 axial. Cancer Sci 109: 2188-2198, 2018.

24. Jiang X, Zhou Y, Sun AJ and Xue JL: NEAT1 contributes to breast cancer progression through modulating miR-448 and ZEB1. J Cell Physiol 233: 8558-8566, 2018.

25. Li R, Fang L, Pu Q, Bu H, Zhu P, Chen Z, Yu M, Li X, Weiland T, Bansal A, et al: MEG3-4 is a miRNA decoy that regulates IL-1 $\beta$ abundance to initiate and then limit inflammation to prevent sepsis during lung infection. Sci Signal 11: pii: eaao2387, 2018.

26. Ye J, Wang C, Wang D and Yuan H: LncRBA GSA5, up-regulated by ox-LDL, aggravates inflammatory response and MMP expression in THP-1 macrophages by acting like a sponge for miR-221. Exp Cell Res 369: 348-355, 2018.

27. Zaynab M, Fatima M, Abbas S, Umair M, Sharif Y and Raza MA Long non-coding RNAs as molecular players in plant defense against pathogens. Microb Pathog 121: 277-282, 2018.

28. Wang K, Long B, Zhou LY, Liu F, Zhou QY, Liu CY, Fan YY and Li PF: CARL IncRNA inhibits anoxia-induced mitochondrial fission and apoptosis in cardiomyocytes by impairing miR-539-dependent PHB2 downregulation. Nat Commun 5: 3596, 2014

29. Zhou X, Zhang W, Jin M, Chen J, Xu W and Kong X: lncRNA MIAT functions as a competing endogenous RNA to upregulate DAPK 2 by sponging miR-22-3p in diabetic cardiomyopathy. Cell Death Dis 8: e2929, 2017.

30. Dangwal S, Schimmel K, Foinquinos A, Xiao K and Thum T: Noncoding RNAs in heart failure. Handb Exp Pharmacol 243: 423-445, 2017.

31. Ma M, Hui J, Zhang QY, Zhu Y, He Y and Liu XJ: Long non-coding RNA nuclear-enriched abundant transcript 1 inhibition blunts myocardial ischemia reperfusion injury via autophagic flux arrest and apoptosis in streptozotocin-induced diabetic rats. Atherosclerosis 277: 113-122, 2018.

32. Su X, Xing J, Wang Z, Chen L, Cui M and Jiang B: microRNAs and ceRNAs: RNA networks in pathogenesis of cancer. Chin J Cancer Res 25: 235-239, 2013.

33. Yao Y, Ma J, Xue Y, Wang P, Li Z, Liu J, Chen L, Xi Z, Teng H, Wang Z, et al: Knockdown of long non-coding RNA XIST exerts tumor-suppressive functions in human glioblastoma stem cells by up-regulating miR-152. Cancer Lett 359: 75-86, 2015. 